\title{
Étude in vivo de l'activité fermentaire cæcale chez le lapin. Mise au point et validation d'une nouvelle technique de canulation cæcale
}

\author{
T Gidenne, R Bellier
}

INRA, laboratoire de recherches sur l'élevage du lapin, BP 27, 31326 Castanet-Tolosan, France

(Reçu le 27 février 1992; accepté le 1er juillet 1992 )

\begin{abstract}
Résumé - Une nouvelle technique de canulation cæcale basée sur l'emploi d'une canule en verre a été mise au point et validée chez le lapin adulte, dans le but d'étudier in vivo, en conditions physiologiques, l'activité fermentaire cæcale. Cette technique n'entraîne pas de modification de digestibilité de la ration et permet de prélever des digesta $(10 \mathrm{~g})$ en quelques minutes en minimisant le stress des animaux, à partir du $10^{\circ}$ jour après opération. Les teneurs en acides gras volatils AGV (60 mM/l) et en ammoniac (11 mM/l) des digesta prélevés in vivo ne diffèrent pas de celles mesurées sur les digesta obtenus après abattage. Les mesures in vivo des paramètres fermentaires sont reproductibles ( $C V=6,8 \%$ pour les $A G V$ totaux) et sont peu influencées par la fréquence quotidienne des prélèvements (1-5 par j). En période préprandiale, les teneurs en AGV sont réduites $(23 \mathrm{mM} / \mathrm{l})$ et le rapport butyrate/acétate est inversé $\left(C_{4} / C_{3}=0,6\right) ; 5 \mathrm{~h}$ après le repas la teneur en AGV est maximale $(96 \mathrm{mM} / \mathrm{l})$, tandis que la proportion de butyrate $(12 \%)$ devient supérieure à celle du propionate $(3,5 \%)$. L'activité fermentaire cæcale d'un lapereau âgé de 7 semaines diffère de celle de l'adulte par une acidité volatile totale plus élevée et une plus forte variation post-prandiale du profil fermentaire.
\end{abstract}

fermentation / cæcum / lapin / canulation / méthodologie

Summary - In vivo study of caecal fermentation in the rabbit. Validation of a new technique for caecal cannulation. A new technique for caecal cannulation in adult rabbit was validated in order to perform in vivo studies of caecal fermentation under a physiological status. The cannulation did not modify the digestibility of the diet, and it was possible to collect digesta (10 g fresh matter) after a few minutes without stress for animals only 10 days post-surgery. Volatile fatty acid (VFA) (60 $\mathrm{mM} / \mathrm{l})$ and ammonia (11 $\mathrm{mM} / \mathrm{l})$ concentration of caecal digesta collected in vivo was not different from that obtained from slaughtered rabbits. VFA measurements were reproducible $(\mathrm{CV}=6.8 \%)$ and were not dependent on the daily collection frequency (1-5/day). Caecal VFA level in the starved rabbit was very low $(23 \mathrm{mM} / \mathrm{l})$ while the butyrate/propionate rate was the inverse $\left(C_{4} / C_{3}=0.6\right)$. Five $h$ after feeding, the in vivo VFA level reached $96 \mathrm{mM} /$ and the butyrate proportion (12\%) over-reached those of propionate (3.5\%). The caecal fermentations of a 7 -week-old rabbit differed from those of an adult by a higher VFA level and by a greater post-feeding variation in the fermentation pattern.

fermentation / caecum / rabbit / cannulation / methodology 


\section{INTRODUCTION}

Le lapin, herbivore monogastrique, possède un système digestif adapté à la dégradation des fibres (un tiers du régime). Le cæcum ( $40 \%$ de la masse digestive totale) est le site d'une importante activité fermentaire comparable à celle du rumen (Cools et Jeuniaux, 1961; Marty et Raynaud, 1966). La composition des digesta parvenant au cæcum influence l'activité de la flore (Candau et al, 1978; Morisse et al, 1985), et une altération de cette dernière peut favoriser l'apparition de troubles digestifs, en particulier chez le lapin en croissance (Peeters et al, 1988). Cependant, peu de travaux ont étudié le contrôle nutritionnel de l'activité fermentaire cæcale chez le lapin.

Les variations des paramètres fermentaires ont généralement été étudiées à partir de digesta prélevés après sacrifice de l'animal; l'information est donc ponctuelle et ne permet pas un contrôle des variations interindividuelles. L'implantation d'une canule cæcale est susceptible de résoudre ces problèmes, en particulier celui de prélèvements en série sur un même individu, dans des conditions physiologiques. Quelques auteurs ont décrit, chez le lapin adulte, des techniques d'implantation dans le cæcum d'une canule en PVC ou en téflon (Yoshida et Kandatsu, 1967; Angelini et al, 1973; Carman et Waynforth, 1984), mais aucun n'a contrôlé la validité de la technique dans le cadre d'études de nutrition.

Notre étude concerne donc la mise au point, chez le lapin adulte, d'une technique de prélèvement in vivo de digesta cæcaux en vue d'étudier l'activité fermentaire. Un travail antérieur concernant la canulation iléale (Gidenne et al, 1988) a permis de montrer l'avantage des canules en verre par rapport aux modèles classiques en matière plastique : meilleure résistance, implantation digestive plus aisée du fait d'une taille réduite, écoulement des digesta facilité. Nous avons donc tout d'abord mis au point une technique de canulation cæcale basée sur l'emploi d'un nouveau type de canule en verre. Puis, nous avons réalisé 3 études méthodologiques afin de contrôler la validité du modèle expérimental proposé, soit :

- étude 1 = contrôle de l'absence de perturbations du système digestif et de la fonctionnalité de la canule;

- étude 2 = comparaison avec la technique classique de prélèvement après abattage, analyse de l'incidence de l'âge des animaux et de l'heure de prélèvement;

- étude 3 = mesure de la reproductibilité des prélèvements et analyse de l'incidence de la fréquence des prélèvements.

\section{MATÉRIEL ET MÉTHODES}

\section{Animaux et alimentation}

Les lapins, de race néozélandaise blanche, sont maintenus en cage à métabolisme dans un local éclairé de $7 \mathrm{~h} 00 \mathrm{à} 19 \mathrm{~h} 00$. L'alimentation est rationnée à raison de $120 \mathrm{~g} / \mathrm{j}$. La composition de l'aliment expérimental est présentée au tableau I.

L'étude $n^{\circ} 1$ porte sur 2 lots de 6 lapins adultes $(2,7-3 \mathrm{~kg}$ de poids vif) : un lot témoin et un lot de lapins porteurs d'une canule cæcale. L'aliment est distribué quotidiennement à $9 \mathrm{~h} 00$.

Lors de l'étude $n^{\circ} 2$, l'aliment expérimental est distribué en 2 repas $(8$ à $11 \mathrm{~h}$ et de 16 à 18 $h)$, dans le but de réduire les variations interindividuelles provenant d'un rythme d'ingestion asynchrone entre les animaux. Trois lots de lapins néozélandais blanc ont été constitués : un lot de 4 lapins adultes porteurs d'une canule cæcale (poids vif moyen $=3010 \pm 200 \mathrm{~g}$ ), un lot de 16 lapins adultes, lot $A$ (poids vif moyen $=$ $3196 \pm 184 \mathrm{~g}$ ), et un lot de 16 lapereaux âgés de 7 semaines, lot $\mathrm{J}$ (poids vif moyen $=1010 \pm$ $76 \mathrm{~g}$ ).

L'étude $n^{\circ} 3$ porte sur un lot de 4 lapins porteurs d'une canule cæcale, recevant quotidien- 
Table I. Composition et analyse chimique de l'aliment expérimental.

\begin{tabular}{lr}
\hline Ingrédients & $(\%)$ \\
\hline & \\
Blé & 37,4 \\
Luzerne déshydratée & 32,0 \\
Son de blé & 10,2 \\
Paille de blé & 6,1 \\
Tourteau de soja & 11,2 \\
Minéraux et vitamines & 3,1 \\
& \\
Composition chimique (\% sec) & \\
& \\
Matière organique & \\
Protéines brutes & 90,8 \\
Cellulose brute & 18,5 \\
NDF & 12,3 \\
ADF & 32,6 \\
Lignine (ADL) & 16,5 \\
& 4,2 \\
\hline
\end{tabular}

nement (à $9 \mathrm{~h} \mathrm{00)}$ une ration de $120 \mathrm{~g}$ d'aliment expérimental.

\section{Technique chirurgicale}

Le modèle de la canule, en verre Pyrex, est décrit dans la figure 1. Une collerette en fibres synthétiques est fixée à la base de la canule afin de permettre le maintien de la canule sur la paroi cæcale et de faciliter le développement d'adhérences séreuses cæco-pariétales. Une seconde pièce en Pyrex est emboitée sur la canule, en vue de réduire les mouvements vers l'intérieur de la cavité abdominale et de renforcer la partie tubulaire de la canule.

Les lapins sont mis à jeun $24 \mathrm{~h}$ avant l'opération. Après anesthésie à l'aide d'un mélange $x y$ lazine-kétamine, et en respectant les conditions classiques d'asepsie, la paroi abdominale est incisée sur 6-7 cm le long de la ligne médiane. La canule est implantée entre la $4^{e}$ et la $5^{e}$ spire du cæcum, après la pose d'une suture en bourse (polyamide, dec 2.0) et incision de la paroi cæcale sur environ $1,5 \mathrm{~cm}$. Le maintien de la canule sur la paroi cæcale est obtenu en serrant la suture en bourse, et en posant 4 sutures
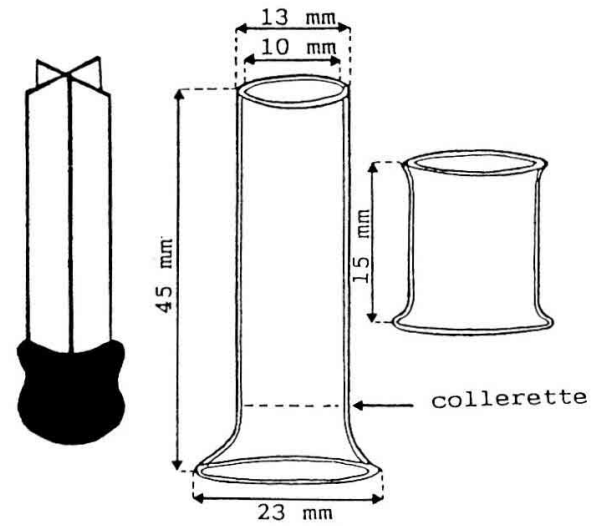

(1)

(2)

(3)

Fig 1. Canule cæcale. (1) Bouchon (matière plastique). (2) Canule (Pyrex). (3) Pièce externe de protection (Pyrex).

simples (polyamide, dec 1.5) entre la collerette et la paroi cæcale. La canule est ensuite extériorisée au niveau du flanc droit de l'animal, puis on procède à la suture des plans musculaires (catgut résorbable, dec 3.0) et cutané (polyester tressé, dec 2.0) de l'abdomen. En dernier lieu, la pièce externe de protection est emboîtée sur la canule. Les animaux reçoivent un traitement antibiotique à base de spiramycine et de colistine pendant les 4 j suivant l'opération.

\section{Technique de prélèvement des digesta}

\section{Prélèvements in vivo}

La technique de prélèvement des digesta est inspirée de celle mise au point antérieurement pour les récoltes de digesta à l'iléon (Gidenne et al, 1988). Les animaux sont placés, en position naturelle de repos, dans un hamac spécialement adapté. Ceci évite une manipulation de l'animal lors de l'écoulement des digesta, et permet de prélever avec aisance et rapidité. Les digesta s'écoulent par gravité; ils sont stockés dans un flacon contenant un agent conservateur 
$\left(\mathrm{H}_{2} \mathrm{SO}_{4}, \mathrm{H}_{3} \mathrm{PO}_{4}\right)$ à $-18^{\circ} \mathrm{C}$. La durée totale d'un prélèvement n'excède pas $15 \mathrm{~min}$, incluant la saisie du lapin, son installation dans le hamac, le prélèvement et la mesure du $\mathrm{pH}$.

\section{Prélèvements après sacrifice de l'animal}

Les prélèvements sont effectués selon la procédure suivante : saisie des lapins, transport à l'abattoir, saisie, électronarcose, saignée, éviscération, mesure du pH, prélèvement. La durée totale des manipulations est d'environ $30 \mathrm{~min}$.

\section{Protocoles de prélèvement}

Lors de l'étude $n^{\circ} 2$, l'évolution post-prandiale des paramètres fermentaires est analysée, à partir de 4 prélèvements : $30 \mathrm{~min}$ avant le repas, et à 5,7 et $9 \mathrm{~h}$ après le début du repas. L'incidence de la technique de prélèvement est étudiée en comparant la qualité des digesta recueillis in vivo sur les lapins canulés (une récolte par jour pendant 4 j consécutifs, sur chaque lapin) à ceux recueillis après le sacrifice de 4 lapins (lot A) par h de prélèvement et par jour. L'activité fermentaire en fonction de l'âge de l'animal est étudiée en comparant la composition des digesta des lots $\mathrm{A}$ et $\mathrm{J}$ (prélèvements après sacrifice).

Lors de l'étude $n^{\circ} 3$, la reproductibilité des prélèvements in vivo est mesurée en comparant la composition d'échantillons récoltés en période d'activité fermentaire élevée ( $7 \mathrm{~h} 30$ après le début du repas), dans les mêmes conditions pendant 4 j consécutifs. L'incidence de la fréquence quotidienne des prélèvements FQP a été étudiée en comparant la composition des échantillons obtenus à $20 \mathrm{~h}$, en fonction du nombre de prélèvements réalisés le même jour entre 8 et $20 \mathrm{~h}(F Q P=1$ à 5$)$. Les prélèvements sont effectués sur une période de 5 jours consécutifs selon la chronologie suivante (répétée une fois en ordre croissant de fréquence de prélèvement) (tableau II).

\section{Mesure de digestibilité apparente fécale}

La digestibilité est mesurée lors de l'étude $n^{\circ} 1$, simultanément pour les animaux témoins et ca-
Table II. Chronologie des prélèvements.

Jour FQP Heures desprélèvements

\begin{tabular}{lllllllll}
$J_{1}$ & 5 & 8 & - & 11 & - & 14 & -17 & -20 \\
$J_{2}$ & 2 & 8 & - & & & & & -20 \\
$J_{3}$ & 3 & 8 & - & & & 14 & & -20 \\
$J_{4}$ & 1 & - & & & & & & -20 \\
$J_{5}$ & 4 & 8 & - & 12 & -16 & & -20 \\
\hline
\end{tabular}

nulés pendant $6 j$ consécutifs ( $4 \mathrm{j}$ après une première collecte de digesta). Chaque jour, la totalité des fèces est récoltée, pesée, puis stockée à $-18{ }^{\circ} \mathrm{C}$ en sachet plastique étanche, en vue de leur analyse ultérieure.

\section{Analyses}

Les analyses suivantes ont été réalisées sur l'aliment et les fèces : matière sèche MS par dessiccation pendant $24 \mathrm{~h}$ à $103^{\circ} \mathrm{C}$, matières minérales après incinération pendant $5 \mathrm{~h}$ à $550^{\circ} \mathrm{C}$, matières azotées totales MAT (méthode Kjeldhal, $N \times 6,25$ ), constituants pariétaux totaux Van-Soest NDF selon la méthode semiautomatique adaptée par Giger et al (1979).

Après décongélation, les échantillons de contenus de cæcum sont centrifugés $(8000 \mathrm{~g})$, les acides gras volatils (AGV) et l'ammoniac $\left(\mathrm{NH}_{3}\right)$ sont dosés sur la fraction surnageante. L'analyse des AGV est réalisée par chromatographie en phase gazeuse selon la technique décrite par Jouany (1982), et adaptée pour le dosage sur colonne semi-capillaire. Le dosage d'ammoniac est réalisé après dialyse, par colorimétrie selon la méthode de Weatherburn (1967) adaptée à l'analyse en flux continu.

\section{Statistiques}

L'analyse statistique des données est réalisèe par analyse de variance en utilisant un modèle linéaire général (programme GLM) de la bibliothèque statistique SAS (1985). 


\section{RÉSULTATS}

\section{Étude 1. Technique de canulation cæcale et incidence sur la digestion}

Dès le lendemain de l'opération, l'ingestion des animaux reprend et revient à son niveau initial environ $4 \mathrm{j}$ après l'opération. La perte de poids post-opératoire, d'environ 200-300 g, est récupérée en une dizaine de jours. Dans nos conditions opératoires, le taux de réussite d'implantation d'une canule fonctionnelle est de $90 \%$; seulement $10 \%$ des animaux ont présenté quelques troubles post-opératoires tels que perte d'appétit ou inflammation locale. Les premiers prélèvements de digesta peuvent être réalisés dès le $10^{\mathrm{e}} \mathrm{j}$ post-opératoire. La quantité de digesta frais obtenue lors d'une récolte de $10 \mathrm{~min}$ est en moyenne de $10 \mathrm{~g}$, soit $8-10 \%$ du contenu cæcal total d'un lapin adulte. La durée totale de manipulation n'excédant pas $15 \mathrm{~min}$, cela permet de minimiser le stress des lapins. Un animal ainsi opéré peut conserver une canule fonctionnelle durant 3-6 mois.

Nous n'observons aucune incidence significative de la canulation sur la digestibilité apparente de la ration (tableau III) quel que soit le nutriment. En particulier, la dégradation des constituants pariétaux (NDF) par la flore cæcale n'est pas affectée par l'implantation d'une canule.

\section{Étude 2. Incidence de la technique et de l'heure de prélèvement sur la composition des digesta}

Les teneurs en ammoniac $\left(\mathrm{NH}_{3}\right)$ et en acides gras volatils totaux (AGV) des prélèvements obtenus in vivo chez l'animal canulé ne diffèrent pas significativement de celles mesurées pour les digesta obtenus sur l'animal abattu (tableau IV); cepen-
Tableau III. Incidence de la canulation sur la digestibilité fécale.

\begin{tabular}{lcccc}
\hline Lot & MS & MO & NDF & MAT \\
\hline $\begin{array}{l}\text { Canulés } \\
(n=6)\end{array}$ & 66,8 & 67,2 & 36,8 & 74,6 \\
$\begin{array}{l}\text { Témoins } \\
(n=6)\end{array}$ & 67,4 & 67,9 & 37,8 & 74,7 \\
$\begin{array}{l}\sigma_{\text {res }} \\
\text { Sign stat }(P<)\end{array}$ & 0,51 & 0,44 & 0,55 & 0,95 \\
\hline
\end{tabular}

dant, nous remarquons une acidité un peu plus forte de ces derniers $(-0,2$ unités $\mathrm{pH})$. La proportion d'acétate $\left(C_{2}\right)$ est plus faible et celle de propionate $\left(\mathrm{C}_{3}\right)$ plus forte, dans les digesta obtenus in vivo; mais ces écarts ne sont significatifs que pour les digesta prélevés chez l'animal en situation de jeûne (prélèvement à $7 \mathrm{~h} 30$, avant le repas).

L'effet de l'heure de prélèvement est significatif, quel que soit le critère mesuré, excepté pour la teneur en matière sèche. Nous observons un pic fermentaire (quadruplement de la teneur en AGV et une réduction de $75 \%$ de la teneur en $\mathrm{NH}_{3}$ cæcal) seulement $5 \mathrm{~h}$ après le début du repas du matin (fig 2). En situation préprandiale le rapport $C_{4} / C_{3}$ (fig 3) est inversé, tandis qu'après le repas les proportions de $C_{4}$ et de $C_{3}$ restent stables et sont celles classiquement observées chez le lapin $\left(C_{4} / C_{3}=2,4\right)$. Par contre, la proportion d'acétate ( $83 \%$ en moyenne) varie très faiblement au cours du temps.

\section{Incidence de l'âge de l'animal sur l'évolution post-prandiale des fermentations cæcales}

La teneur en MS du contenu cæcal d'un lapereau de 7 semaines est inférieure à 
Tableau IV. Incidence de la technique et de l'heure de prèlèvement sur la composition du contenu cæcal.

\begin{tabular}{|c|c|c|c|c|c|c|c|}
\hline Technique & $\begin{array}{l}M S \\
(\%)\end{array}$ & $p H$ & $\begin{array}{c}\mathrm{NH}_{3} \\
(\mathrm{mMI})\end{array}$ & $\begin{array}{c}A G V \\
(m M I)\end{array}$ & $\begin{array}{l}C_{2} \\
(\%)\end{array}$ & $\begin{array}{l}C_{3} \\
(\%)\end{array}$ & $\begin{array}{l}C_{4} \\
(\%)\end{array}$ \\
\hline Canule a & 23,1 & 6,33 & 10,0 & 58,2 & 83,9 & 5,6 & 8,4 \\
\hline Abattage ${ }^{a}$ & 22,7 & 6,13 & 12,0 & 63,2 & 86,4 & 3,6 & 8,3 \\
\hline$\sigma_{\text {res }}$ & 1,1 & 0,2 & 3,7 & 6,8 & 2,5 & 1,0 & 1,9 \\
\hline \multicolumn{8}{|l|}{ Sign stat } \\
\hline Technique & 0,77 & 0,02 & 0,17 & 0,50 & 0,02 & 0,001 & 0,60 \\
\hline Heure de prélèvement & 0,20 & 0,001 & 0,001 & 0,001 & 0,014 & 0,002 & 0,001 \\
\hline Interaction & 0,74 & 0,40 & 0,25 & 0,047 & 0,048 & 0,002 & 0,50 \\
\hline
\end{tabular}

a Moyennes générales de 16 répétitions ( 4 échantillons $\times 4 \mathrm{~h}$ de prélèvements).

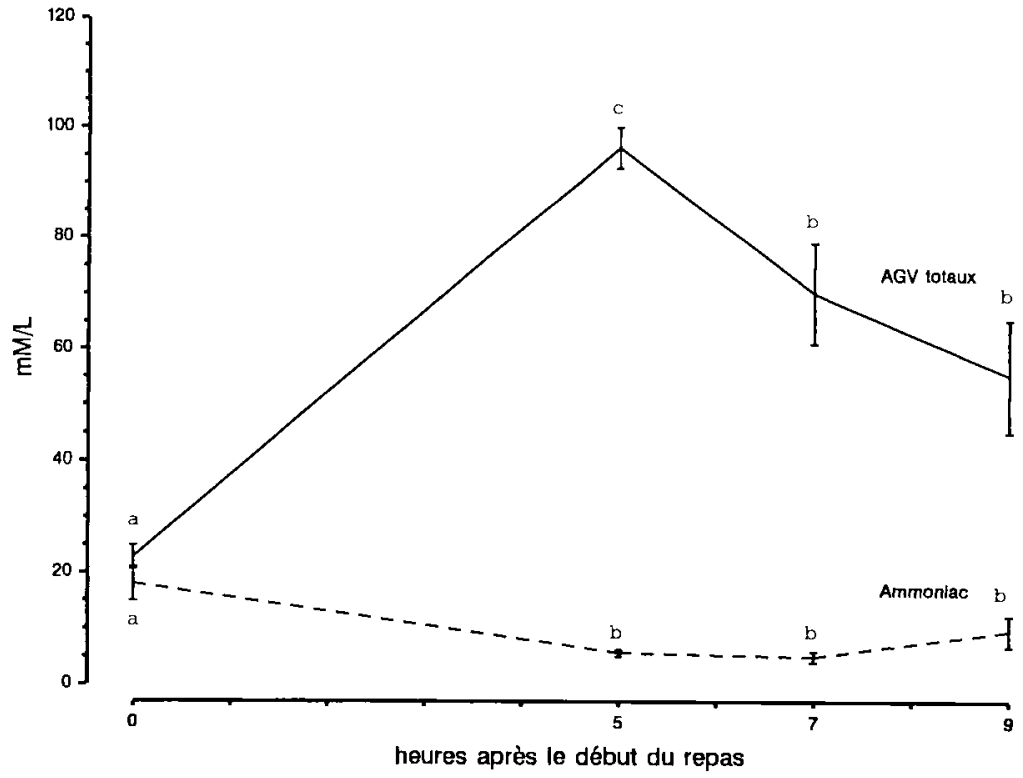

Fig 2. Évolution post-prandiale des concentrations cæcales in vivo, acides gras, volatils et en ammoniac, chez le lapin adulte. Valeurs moyennes issues de mesures sur 4 lapins canulés. a,b Les points moyens affectés d'une même lettre ne diffèrent pas au seuil $P=0,05$.

celle des lapins adultes $(20,8$ vs $22,7 \%$ en moyenne), sans variations significatives en fonction de l'heure. Comparées aux te- neurs observées chez l'adulte, les teneurs en AGV sont plus élevées et les teneurs en $\mathrm{NH}_{3}$ sont plus faibles chez le lapereau 

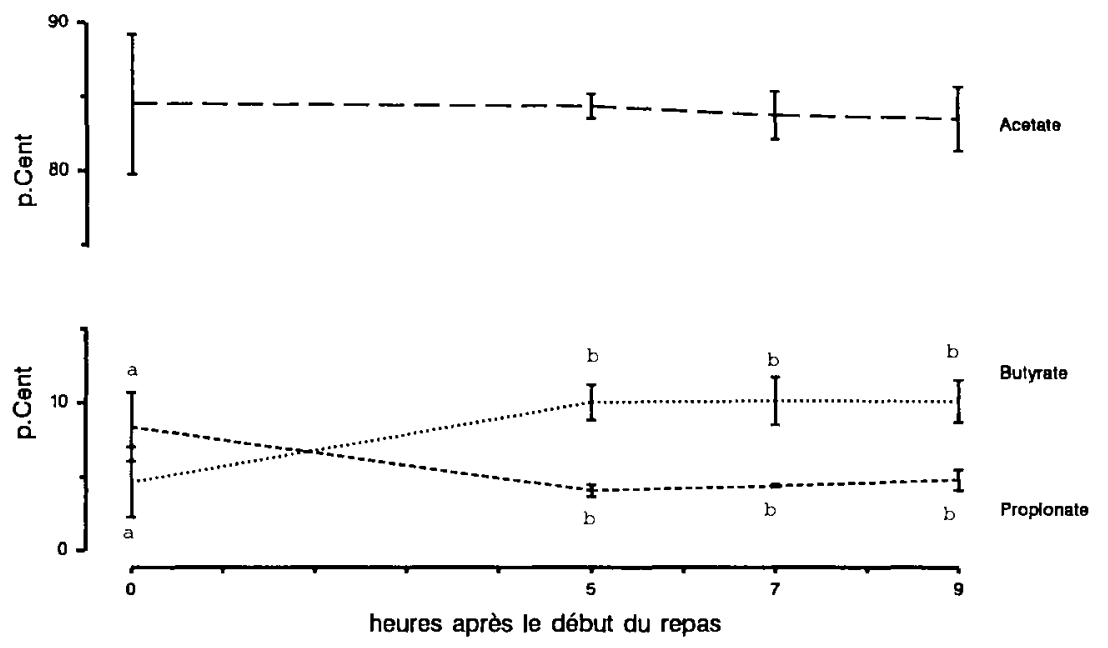

Fig 3. Ėvolution post-prandiale des proportions molaires des acides gras volatils cæcaux, chez le lapin adulte. Valeurs moyennes issues de mesures sur 4 lapins canulés. $a, b$ Les points moyens affectés d'une même lettre ne diffèrent pas au seuil $P=0,05$.

(tableau V). Ces écarts, importants chez l'animal à jeun, se réduisent après le repas du matin, et s'inversent $9 \mathrm{~h}$ plus tard. Les proportions molaires des AGV varient également en fonction de l'âge, plus particulièrement dans le cas du $\mathrm{C}_{3}$ et du $\mathrm{C}_{4}$. Ainsi après le repas, le rapport $\mathrm{C}_{4} / \mathrm{C}_{3}$ est en moyenne de 4,0 chez le lapereau contre 2,9 chez l'adulte. De plus, avant le repas, nous n'observons pas chez le lapereau une inversion du rapport $\mathrm{C}_{4} / \mathrm{C}_{3}$.

\section{Étude 3. Reproductibilité et incidence de la fréquence des prélèvements sur la qualité des échantillons}

Pour un prélèvement obtenu en période d'activité fermentaire élevée (16 h 30), nous n'observons aucun effet du jour de prélèvement (sur $4 \mathrm{j}$ consécutifs) quel que soit le critère considéré (tableau VI). Nous observons par contre un effet très signifi- catif de l'individu en ce qui concerne les teneurs (CV interindividus $=11,3 \%$ ) et les proportions en AGV. La variabilité interjours des teneurs en AGV totaux atteint $5,7 \%$; si elle est cumulée aux variations résiduelles le coefficient de variation total des mesures est de $6,8 \%$. Par contre, la variabilité des mesures de teneurs en $\mathrm{NH}_{3}$ atteint $35 \%$ (CV interindividus $=53 \%$ ). Plus concrètement, la précision de notre méthode permet de mettre en évidence $(P<$ $0,05)$ un écart de $15 \%$ entre les teneurs en AGV totaux, avec seulement 5 répétitions de la mesure.

L'augmentation du nombre de prélèvements quotidiens de 1 à 5 n'influence pas significativement la composition des digesta recueillis à $20 \mathrm{~h}$ (tableau VII), excepté leur teneur en MS. Nous observons ainsi une réduction progressive du taux de MS des contenus cæcaux, accentuée pour la fréquence maximale de prélèvements. Cependant la réalisation de plus de 3 prélèvements par jour entraîne une nervosité 
Tableau V. Évolution post-prandiale du profil fermentaire cæcal en fonction de l'âge du lapin. A : digesta récoltés après sacrifice de 4 lapins adultes, à chaque heure de prélèvements. $J$ : digesta récoltés après sacrifice de 4 lapins âgés de 7 semaines, à chaque heure de prélèvements.

\begin{tabular}{|c|c|c|c|c|c|c|c|}
\hline $\begin{array}{l}\text { Heures après } \\
\text { le repas }\end{array}$ & Age & $p H$ & $\begin{array}{c}\mathrm{NH}_{3} \\
\left.(\mathrm{mM} /)^{\prime}\right)\end{array}$ & $\begin{array}{c}A G V \\
(m M I)\end{array}$ & $\begin{array}{l}C_{2} \\
(\%)\end{array}$ & $\begin{array}{l}C_{3} \\
(M)\end{array}$ & $\begin{array}{l}C_{4} \\
(\%)\end{array}$ \\
\hline 0 & $\begin{array}{l}A \\
J\end{array}$ & $\begin{array}{l}6,50 \\
6,19\end{array}$ & $\begin{array}{l}24,4^{\mathrm{a}} \\
11,5^{\mathrm{b}}\end{array}$ & $\begin{array}{l}35,8^{b} \\
54,5^{a}\end{array}$ & $\begin{array}{l}91,4 \\
87,3\end{array}$ & $\begin{array}{l}3,7 \\
3,3\end{array}$ & $\begin{array}{l}3,0^{\mathrm{b}} \\
8,4^{\mathrm{a}}\end{array}$ \\
\hline 5 & $\begin{array}{l}A \\
J\end{array}$ & $\begin{array}{l}5,95^{\mathrm{a}} \\
5,47^{\mathrm{b}}\end{array}$ & $\begin{array}{l}9,7 \\
8,2\end{array}$ & $\begin{array}{r}88,1^{\mathrm{a}} \\
107,1^{\mathrm{b}}\end{array}$ & $\begin{array}{l}84,0 \\
83,8\end{array}$ & $\begin{array}{l}3,7 \\
2,7\end{array}$ & $\begin{array}{l}11,0 \\
12,7\end{array}$ \\
\hline 7 & $\begin{array}{l}A \\
J\end{array}$ & $\begin{array}{l}5,96 \\
5,86\end{array}$ & $\begin{array}{l}6,2 \\
6,5\end{array}$ & $\begin{array}{l}69,2^{\mathrm{b}} \\
80,9^{\mathrm{a}}\end{array}$ & $\begin{array}{l}84,1 \\
84,5\end{array}$ & $\begin{array}{l}3,3 \\
2,7\end{array}$ & $\begin{array}{l}10,6 \\
11,2\end{array}$ \\
\hline 9 & $\begin{array}{l}A \\
J\end{array}$ & $\begin{array}{l}6,09 \\
6,10\end{array}$ & $\begin{array}{l}7,7 \\
9,3\end{array}$ & $\begin{array}{l}58,5^{\mathrm{a}} \\
50,6^{\mathrm{b}}\end{array}$ & $\begin{array}{l}86,0 \\
83,7\end{array}$ & $\begin{array}{l}3,7 \\
3,3\end{array}$ & $\begin{array}{r}8,9 \\
10,6\end{array}$ \\
\hline$\sigma_{\text {res }}$ & & 0,22 & 3,7 & 6,7 & 2,7 & 0,5 & 2,4 \\
\hline $\begin{array}{l}\text { Sign stat } \\
\text { Age } \\
\text { Heure } \\
\text { Interaction }\end{array}$ & & $\begin{array}{l}0,011 \\
0,001 \\
0,16\end{array}$ & $\begin{array}{l}0,032 \\
0,001 \\
0,003\end{array}$ & $\begin{array}{l}0,001 \\
0,001 \\
0,003\end{array}$ & $\begin{array}{l}0,13 \\
0,002 \\
0,35\end{array}$ & $\begin{array}{l}0,001 \\
0,16 \\
0,35\end{array}$ & $\begin{array}{l}0,016 \\
0,001 \\
0,27\end{array}$ \\
\hline
\end{tabular}

a.b Pour une même heure de prélèvement, les moyennes affectées d'une même lettre ne diffèrent pas au seuil $P=$ 0,05 .

plus marquée des animaux lors des récoltes de digesta, sans entrainer toutefois une réduction de l'ingestion.

\section{DISCUSSION}

\section{Impact de la canulation cæcale chez le lapin adulte}

Par rapport aux techniques antérieures (canules en PVC ou en téflon), l'emploi d'une canule en verre Pyrex permet de réduire notablement les complications post-opératoires. Le rétablissement des animaux est très rapide; ainsi il est possible de réaliser les premiers prélèvements seulement $10 \mathrm{j}$ après l'opération, tandis que Carman et Waynforth (1984) signalent un délai d'attente de $30 \mathrm{j}$ minimum. La viabilité des animaux canulés ( 3 mois minimum) est compatible avec la durée d'une étude de nutrition, et l'implantation d'une canule cæcale n'a pas de conséquences sur la digestibilité. En comparaison, la pose d'une canule iléale, plus délicate et plus traumatisante pour l'animal, n'avait pas non plus d'effet sur l'utilisation digestive de la ration (Kametaka, 1970; Gidenne et Ruckebusch, 1989). 
Tableau VI. Reproductibilité de la composition du contenu cæcal prélevé in vivo.

\begin{tabular}{|c|c|c|c|c|c|c|c|}
\hline Jour & $\begin{array}{l}M S \\
(\%)\end{array}$ & $p H$ & $\begin{array}{c}\mathrm{NH}_{3} \\
(\mathrm{mMl})\end{array}$ & $\begin{array}{l}A G V \\
(m M I)\end{array}$ & $\begin{array}{l}C_{2} \\
(\%)\end{array}$ & $\begin{array}{c}C_{3} \\
(\%)\end{array}$ & $\begin{array}{c}C_{4} \\
(\%)\end{array}$ \\
\hline $\begin{array}{l}J_{1} \\
J_{2} \\
J_{3} \\
J_{4}\end{array}$ & $\begin{array}{l}21,3 \\
21,2 \\
21,5 \\
21,3\end{array}$ & $\begin{array}{l}5,66 \\
5,68 \\
5,65 \\
5,75\end{array}$ & $\begin{array}{l}4,6 \\
5,1 \\
4,1 \\
5,2\end{array}$ & $\begin{array}{l}114,6 \\
120,0 \\
116,5 \\
109,1\end{array}$ & $\begin{array}{l}83,3 \\
82,8 \\
82,3 \\
82,8\end{array}$ & $\begin{array}{l}3,7 \\
3,7 \\
3,9 \\
3,9\end{array}$ & $\begin{array}{l}12,6 \\
12,4 \\
13,0 \\
12,7\end{array}$ \\
\hline$\sigma_{\text {res }}$ & 0,4 & 0,10 & 1,4 & 4,4 & 1,2 & 0,3 & 1,0 \\
\hline $\begin{array}{l}\text { Sign stat } \\
\text { Jour } \\
\text { Individu }\end{array}$ & $\begin{array}{l}0,62 \\
0,001\end{array}$ & $\begin{array}{l}0,44 \\
0,18\end{array}$ & $\begin{array}{l}0,77 \\
0,09\end{array}$ & $\begin{array}{l}0,17 \\
0,009\end{array}$ & $\begin{array}{l}0,90 \\
0,02\end{array}$ & $\begin{array}{l}0,39 \\
0,06\end{array}$ & $\begin{array}{l}0,83 \\
0,005\end{array}$ \\
\hline
\end{tabular}

Valeurs moyennes $(n=4)$ issues d'une mesure individuelle sur un lot de 4 lapins porteurs de canule cæcale.

Tableau VII. Incidence de la fréquence quotidienne des prélèvements (FQP) sur la composition du contenu cæcal. Valeurs moyennes $(n=8)$ issues de 2 mesures individuelles sur un lot de 4 lapins porteurs de canule cæcale. FQP : nombre de prélèvements quotidiens, pour un même lapin, entre 8 et $20 \mathrm{~h}$.

\begin{tabular}{|c|c|c|c|c|c|c|c|}
\hline$F Q P$ & $\begin{array}{l}M S \\
(\%)\end{array}$ & $p H$ & $\begin{array}{c}\mathrm{NH}_{3} \\
(\mathrm{mM})\end{array}$ & $\begin{array}{c}A G V \\
(m M)\end{array}$ & $\begin{array}{c}C_{2} \\
(\%)\end{array}$ & $\begin{array}{l}C_{3} \\
\text { (\%) }\end{array}$ & $\begin{array}{l}C_{4} \\
(\%)\end{array}$ \\
\hline 1 & $21,5^{b}$ & 6,00 & 5,1 & 94,8 & 83,0 & 4,4 & 11,7 \\
\hline 2 & $21,4^{b}$ & 6,07 & 3,5 & 89,8 & 82,6 & 4,6 & 11,9 \\
\hline 3 & $20,7^{b}$ & 6,12 & 4,0 & 81,8 & 81,8 & 5,0 & 12,3 \\
\hline 4 & $20,6^{b}$ & 6,18 & 3,8 & 85,6 & 83,1 & 4,7 & 11,3 \\
\hline 5 & $19,6^{a}$ & 6,10 & 4,8 & 98,5 & 81,9 & 4,6 & 12,7 \\
\hline$\sigma_{\text {res }}$ & 0,8 & 0,16 & 1,2 & 9,2 & 1,6 & 0,4 & 1,3 \\
\hline \multicolumn{8}{|l|}{ Sign stat } \\
\hline FQP & 0,002 & 0,14 & 0,11 & 0,31 & 0,69 & 0,36 & 0,61 \\
\hline Individu & 0,15 & 0,001 & 0,035 & 0,006 & 0,23 & 0,73 & 0,21 \\
\hline
\end{tabular}

${ }_{a, b}$ Les moyennes affectées d'une même lettre ne diffèrent pas au seuil $P=0,05$.

Validité de la technique de prélèvement

La comparaison des prélèvements obtenus in vivo sur l'animal canulé avec ceux obtenus par abattage ne révèle pas de différences majeures de composition des digesta cæcaux. Quelques écarts ont cependant été remarqués au niveau des 
proportions d'acétate et de propionate des digesta prélevés avant le repas. Une erreur d'échantillonnage pourrait être à l'origine de ces écarts, du fait d'un volume cæcal plus faible en phase pré-prandiale. Toutefois, il est raisonnable de penser que par comparaison à un prélèvement in vivo, la séquence de manipulations préalables à un prélèvement sur l'animal abattu (cf technique de prélèvement) ne peut qu'être préjudiciable à la qualité des échantillons.

Par ailleurs, nous avons constaté qu'une élévation de la fréquence quotidienne des prélèvements modifie peu la qualité des échantillons. Compte tenu de cette bonne reproductibilité, notre méthode paraît convenir pour des études in vivo de cinétiques fermentaires. Toutefois, il paraît souhaitable de ne pas dépasser 3 prélèvements quotidiens afin d'éviter une nervosité excessive des animaux lors de leur manipulation. Une diminution de la quantité de digesta récoltée réduirait le temps de prélèvement et le stress des animaux; il serait alors possible d'augmenter la fréquence quotidienne des prélèvements.

D'autre part, une des finalités de ce travail méthodologique était de connaître les limites d'application de notre modèle expérimental, en relation avec l'âge des animaux. Or, nous constatons un effet important de l'âge du lapin sur les paramètres fermentaires cæcaux : acidité volatile totale et rapport $\mathrm{C}_{4} / \mathrm{C}_{3}$ plus élevés chez le lapereau. De plus, nous observons une sensibilité aux conditions d'alimentation différente entre le lapereau et l'adulte, en particulier en période pré-prandiale. Par ailleurs, les importantes variations post-prandiales des teneurs en produits de la fermentation constatées chez le lapereau suggèrent un métabolisme plus actif au niveau de la flore cæcale. En conséquence, les résultats que l'on obtiendrait à partir de lapins adultes canulés ne pourraient être transposables au lapereau. Mais, du fait de sa matière (verre), le modèle de canule employé ici présente l'avantage de pouvoir être facilement réduit; son adaptation pour le jeune lapin ne devrait pas, en principe, poser de problèmes majeurs, sous peine de réaliser quelques études supplémentaires (chirurgie, technique de prélèvement...). Nous disposerions alors d'un modèle expérimental pour l'étude in vivo de l'activité fermentaire à un stade physiologique délicat qui correspond à une phase d'installation de la flore cæcale.

\section{Évolution post-prandiale des paramètres fermentaires}

Les teneurs en produits de la fermentation dans le cæcum évoluent rapidement après le repas : acidité volatile totale quadruplée et ammoniac cæcal réduit de $75 \%$. Des résultats similaires ont également été constatés par Susmel et Lanari (1976) et par Fioramonti et Ruckebusch (1976). II faut cependant remarquer que les proportions molaires des AGV ne varient pas sensiblement après le repas, mais qu'elles sont profondément modifiées chez l'animal à jeun : le profil classiquement observé chez le lapin $\left(\mathrm{C}_{2}\right.$ majoritaire suivi du $\mathrm{C}_{4}$ puis du $C_{3}$ ) est inversé au niveau du rapport $C_{4} /$ $\mathrm{C}_{3}$. Ce phénomène semble essentiellement provenir de variations de teneurs en butyrate en accord avec les résultats de Vernay et Raynaud (1975). II faut souligner, chez le lapin, le rôle particulier du butyrate comme substrat énergétique préférentiel de l'entérocyte (Vernay, 1987), son taux de production et son absorption (passage actif) différant de ceux de l'acétate et du propionate (Vernay et Marty, 1984). 


\section{CONCLUSION}

Notre technique de canulation et de prélèvement in vivo de digesta cæcaux chez le lapin adulte se caractérise par une absence de conséquences notables pour l'animal, une rapidité et une aisance de prélèvement, la longue durée de vie d'une canule fonctionnelle, la bonne reproductibilité de l'échantillonnage et la possibilité d'effectuer des prélèvements en série. En conséquence, notre modèle expérimental se révèle bien adapté à l'étude des variations nutritionnelles de l'activité fermentaire cæcale, in vivo, chez un animal qui est dans des conditions physiologiques.

Étant donné les différences marquées d'activité fermentaire en fonction de l'âge, les conclusions obtenues à partir du lapin adulte canulé ne seraient pas transposables au lapin plus jeune en phase de post-sevrage. Une étude supplémentaire s'avère donc nécessaire pour adapter notre technique au lapereau.

\section{REMERCIEMENTS}

Nous remercions $M$ Bouillier-Oudot (ENSA Toulouse), Mme Vernay (université PaulSabatier de Toulouse) pour leurs conseils techniques et scientifiques, et A Lapanouse (INRA) pour les soins apportés aux animaux. Cette étude a été réalisée avec la participation financière de la société Duquesne-Purina France.

\section{RÉFÉRENCES}

Angelini A, Cioffi LA, Proto V (1973) Fistolizzazione del cieco e ciecoctomia nel coniglio. Boll Soc It Biol Sper 49, 1390-1396

Candau M, Delpon G, Fioramonti J (1978) Influence de la nature des glucides membranaires sur le développement anatomofonctionnel du tractus digestif du lapin. $2^{\text {es }}$ Journées de la recherche cunicole (1978) Toulouse, communication $n^{\circ} 1$

Carman RJ, Waynforth HB (1984) Chronic fistulation and cannulation of the rabbit caecum. Lab Anim 18, 258-260

Cools A, Jeuniaux C (1961) Fermentation de la cellulose et absorption des AGV au niveau du caecum du lapin. Arch Int Physiol Biochim $69,1-8$

Fioramonti J, Ruckebusch Y (1976) La motricité caecale chez le lapin. 3. Dualité de l'excrétion fécale. Ann Rech Vét 7, 281-295

Gidenne T, Ruckebusch Y (1989) Flow and rate of passage studies at the ileal level in the rabbit. Reprod Nutr Dev 29, 403-412

Gidenne T, Bouyssou T, Ruckebusch $Y$ (1988) Sampling of digestive contents by ileal canulation in the rabbit. Anim Prod 46, 147-151

Giger S, Sauvant D, Dorleans M, Morand-Fehr $P$ (1979) Détermination semi-automatique des constituants membranaires des aliments concentrés par la méthode de Van-Soest. 30th Annual meeting of the EAAP, Harrogate, England, July 1979; Commision on animal feeding, N3.5

Jouany JP (1982) Dosage des acides gras volatils (AGV) et des alcools, dans les contenus digestifs, les jus d'ensilage, les cultures bactériennes et les contenus de fermenteurs anaérobies. Sci Alim 2, 131-144

Kametaka M (1970) Studies on the digestion in the rabbit. XII. Effects of intestinal fistulae fixation on digestibility and passage of radioactive manganese dioxide through the intestinal tract. Agric Biol Chem 34, 12101219

Marty J, Raynaud P (1966) Étude de l'acidité organique au niveau du tube digestif du lapin. Arch Sci Physiol 20, 515-524

Morisse JP, Boilletot E, Maurice R (1985) Alimentation et modifications du milieu intestinal chez le lapin ( $\mathrm{AGV}, \mathrm{NH}_{3}, \mathrm{pH}$, flore). Rec Med Vet 161, 443-449

Peeters JE, Maertens L, Vroonen C, Geeroms R (1988) Influence of spontaneous rotavirus infection on zootechnical performance and caecal parameters in quickly growing commercial weanling rabbits. Deutsche Veterinarmedizinische Gesselschaft, 2-4 juin 1988, Celle, 249-257 
SAS (1985) SAS/STAT. Guide for personal computers, version 6. SAS Institute INC, Cary, NC, $378 p$

Susmel $P$, Lanari $D$ (1976) Changes in volatile fatty acid level in rabbit caecum. $1^{\text {er }}$ congrès int cunicole, Dijon, comm 32

Vernay M (1987) Effects of plasma aldosterone on butyrate absorption and metabolism in the rabbit proximal colon. Comp Biochem Physiol 86, 657-662

Vernay M, Raynaud P (1975) Répartitions des acides gras volatils dans le tube digestif du lapin domestique. 2. Lapins soumis au jeûne. Ann Rech Vét 6, 369-377

Vernay M, Marty J (1984) Absorption and metabolism of butyric acid in rabbit hind-gut. Comp Biochem Physiol 77, 89-96

Weatherburn MW (1967) Phenol-hypochlorite reaction for determination of ammonia. Anal Chem 39, 971-974

Yoshida T, Kandatsu M (1967) Studies on cecum digestion. 7. On the operation for cecum fistula in rabbit and excretion of orally administered $\mathrm{Cr}_{2} \mathrm{O}_{3}$ to hard and soft feces. Jpn J Zootech Sci 38, 358-363 